\title{
4D Shape Registration for Dynamic Electrophysiological Cardiac Mapping
}

\author{
Kevin Wilson ${ }^{1,5}$, Gerard Guiraudon ${ }^{4,5}$, Doug Jones ${ }^{3,4}$, and Terry M. Peters ${ }^{1,2,5}$ \\ ${ }^{1}$ Biomedical Engineering Program \\ ${ }^{2}$ Department of Medical Biophysics \\ ${ }^{3}$ Department of Medicine, The University of Western Ontario \\ ${ }^{4}$ Canadian Surgical Technology and Advanced Robotics (CSTAR) \\ ${ }^{5}$ Imaging Research Labs, Robarts Research Institute, London ON, Canada N6A 5K8 \\ \{kwilson, tpeters\}@imaging.robarts.ca
}

\begin{abstract}
Registration of 3D segmented cardiac images with tracked electrophysiological data has been previously investigated for use in cardiac mapping and navigation systems. However, dynamic cardiac 4D (3D + time) registration methods do not presently exist. This paper introduces two new $4 \mathrm{D}$ registration methods based on the popular iterative closest point (ICP) algorithm that may be applied to dynamic 3D shapes. The first method averages the transformations of the 3D ICP on each phase of the dynamic data, while the second finds the closest point pairs for the data in each phase and performs a least squares fit between all the pairs combined. Experimental results show these methods yield more accurate transformations compared to using a traditional 3D approach (4D errors: Translation $0.4 \mathrm{~mm}$, Rotation $0.45^{\circ}$ vs. 3D errors: Translation $1.2 \mathrm{~mm}$, Rotation $1.3^{\circ}$ ) while also increasing capture range and success rate.
\end{abstract}

\section{Introduction}

Cardiac mapping and navigation systems have been evolving over the past several years and numerous systems now exist that can relate cardiac electrophysiological (EP) data to a spatial coordinate system [1]. These systems create a point cloud of spatial locations of EP data samples from a cardiac chamber, by tracking an EP probe using various types of tracking systems. From this point cloud of data a 3D surface model can be formed to represent the chamber and build a map representing electrical activation on the heart surface. More recently, studies have begun to investigate how pre-operative CT/MR images can be segmented and then registered to the cardiac navigation environment [2]. These surfaces can then be laid over the cardiac map to place the EP data in the context of patientspecific anatomy, as in the Biosense-Webster CARTOMERGE ${ }^{\mathrm{TM}}$ System.

Several fundamental problems exist with these systems. First, the image environment is static, which leads the surgeon to estimate the current position of the tools in the operating room's dynamic environment. In addition, the shape of the EP map has typically been based only on collected data locations. A more 
sophisticated approach has been demonstrated by Holmes et al. who 'paint' the $\mathrm{CT} / \mathrm{MR}$ volume rendering with a colour-wash representation of the acquired EP data 3]. Finally, vital information, such as the frequency spectra of the EP data, which is often important in the study of diseases such as atrial fibrillation, is missing from the cardiac map.

To resolve these problems we have extended the static 3D cardiac mapping environment into one that takes full advantage of 4D (dynamic 3D) cardiac data. As described in our previous work [4, pre-operative 4D CT/MR scans of the patient are collected to create a dynamic heart model. Once in the operating room the EP data can be collected at a sampling rate sufficiently high to capture the inherent frequency components of the signal, as well as associating a spatial location with each data point. At this stage, we have a patient-specific dynamic heart model as well as a dynamic point cloud of the locations of EP data collected in the operating room or EP laboratory.

In this paper, we introduce two novel $4 \mathrm{D}$ shape registration methods to replace the standard 3D iterative closest point (ICP) [5], with a more accurate and robust registration of $4 \mathrm{D}$ shape environments. An evaluation study is then presented to show how using either of the new methods results in a closer approximation of the transformation between the coordinate systems of the source and the target, as well as increasing the success rate of the algorithm with fewer data samples than are necessary for the standard static ICP approach.

\section{Methods}

\subsection{Iterative Closest Point Algorithm}

Besl and McKay [5] introduced a method (iterative closest point (ICP)) for registration of $3 \mathrm{D}$ shapes, which became the standard approach for registering surface objects. The algorithm begins by defining the model shape (or target) as $X$ and the data shape (or source) as $P$. The data shape is then decomposed into a point set. For each point in $P$ the closest point in $X$ is found:

$$
Y=C(P, X)
$$

where $C$ is the closest point operator and $Y$ represents the resulting closest points. From the pairs in $Y$ and $P$, a least squares fit is performed according to [6] in order to obtain a transformation $T$, consisting of a rotation $R$, and a translation $t$ :

$$
T(R, t)=\min \sum d(Y, T(P))^{2}
$$

where $d$ is the distance between point pairs, and $T(P)$ is the result of mapping the points in $P$ with the transformation $T$. After this transformation is applied, the process of finding the closest point pairs, and calculating a new transformation is repeated until a convergence criterion is satisfied.

While many variations of the standard ICP have been developed [789], none have yet been expanded into a dynamic environment. 


\subsection{Average Iterative Closest Point Algorithm}

Consider a 4D anatomical model and a corresponding dynamic EP data set. Typically, these 4D models consist of a series of individual 3D surfaces, and for our cardiac model, each individual 3D shape (extracted from a dynamic CT or MR data set) represents a different phase of a single heartbeat (see Fig. 11). For the data model, we grouped the collected locations from the tracking system into $\mathrm{N}$ bins (the number of phases for which we have created models) based on the phase of the heart at the time each point was acquired. In an ideal situation, we would now have N 3D models and related EP data sets with identical transformations that must be registered to one another.

The first method we refer to as the average iterative closest point algorithm (A-ICP) (see Fig. 21), which treats each phase (model and data) as an individual 3D ICP problem and finds an ideal transformation, $T i$, for each of them. Then to find the unified transformation, Tave, we take the mean of all $T i$ 's in which the

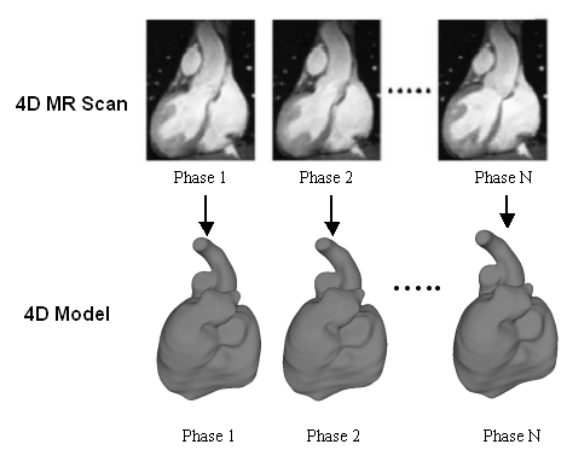

Fig. 1. A dynamic CT or MR scan is acquired, and from each phase image a subjectspecific heart model is generated

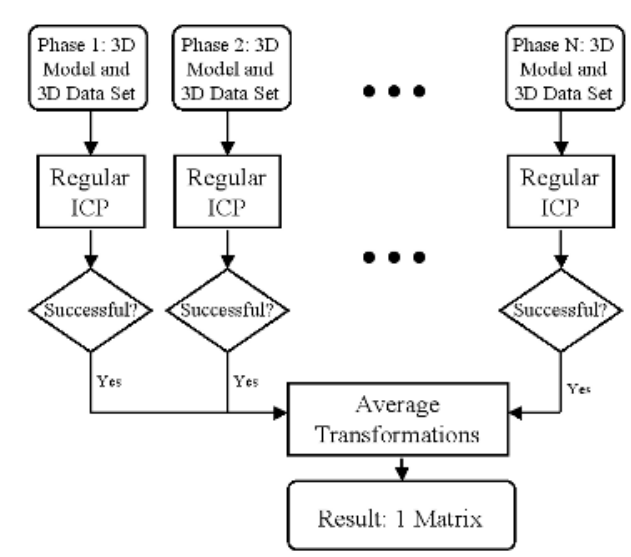

Fig. 2. Flowchart representing average iterative closest point algorithm 
registration was successful (as defined by the success criterion discussed below). To calculate the average transformation we used the quaternion-based mean [10] as a rapid and accurate approximation.

\subsection{Multibin Iterative Closest Point Algorithm}

The second method, the multibin iterative closest point algorithm (M-ICP) (see Fig. 3), groups the 4D model and data into separate bins based on the phase of the heartbeat, as described for the A-ICP approach. Next, we find the closest point pairs between the target model and the data set within each phase (similar to the first stage of the standard 3D ICP). However, once these pairs are found, a least squares fit is performed over all phases combined to obtain a single transform, $T$. This transform is then applied to the data set from each cardiac phase, and the process is repeated until convergence is achieved.

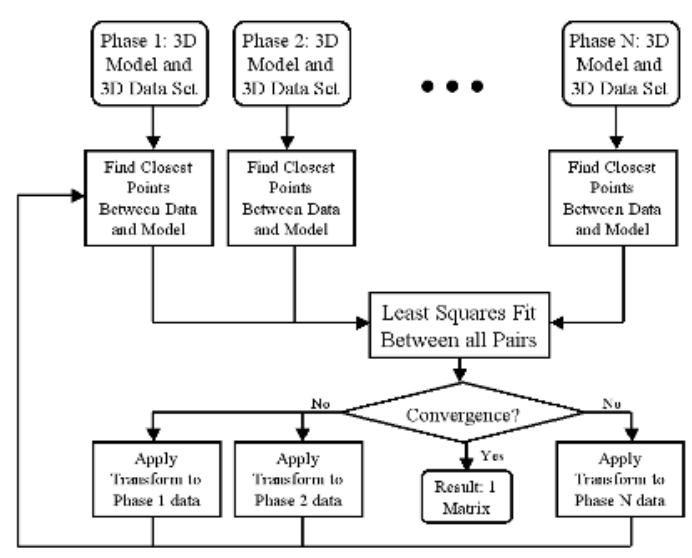

Fig. 3. Flowchart representing multibin iterative closest point algorithm

\section{$3 \quad$ Experiments}

\subsection{Methodology}

To test the accuracy, capture range, and success rate of our algorithms we ran a series of experiments and compared the results provided by the standard ICP, A-ICP, and M-ICP registration algorithms. For our target model we used a 4D heart model consisting of 10 cardiac phases extracted from a high quality 4D MR volume [11. For our data model, we generated point clouds representing samples of EP activity on the cardiac surface as described below.

Regular 3D ICP Registration. The 3D registration experiment was conducted using one of the volumes extracted from our 4D dynamic segmented heart model. From this model, random locations were sampled directly from the cardiac surface to create a data point cloud. Random Gaussian noise was added 
to the coordinates of each data point independently. When generating the noise a standard deviation of $1.5 \mathrm{~mm}$ and mean of 0 was used to represent inaccuracies in the location measurement due to the following errors that were measured in our laboratory using a realistic beating heart phantom, (The Chamberlain Group. Great Barrington, MA., www.thecgroup.com).

- Tracking System Errors $( \pm 1 \mathrm{~mm})$

- Segmentation Errors $( \pm 0.5 \mathrm{~mm})$

- Soft Tissue Deformation $( \pm 1 \mathrm{~mm})$

4D Average and 4D Multibin ICP Registration. Both 4D registration experiments were conducted using all ten phases of our segmented heart model. From this model random locations were sampled directly from the initial phase of the cardiac surface to create a data point cloud. These same locations were then extracted from the dynamic model for phases 2-10. Random Gaussian noise (reflecting the errors cited above) was added independently to each data point as it was for the 3D ICP registration experiment.

The data sets were then transformed with a known rotation and translation according to the experimental parameters (see Table 1). Each experiment was repeated 100 times, and all were conducted with different randomly sampled points with random noise added.

Table 1. 48 separate experiments were each carried out 100 times. Each experiment varied the number of samples in the data set, and the initial rotation and translation the data set was transformed.

\begin{tabular}{|c|c|c|c|c|c|}
\hline \multirow{2}{*}{$\begin{array}{r}\text { Number } \\
\text { of Samples }\end{array}$} & \multirow{2}{*}{$\begin{array}{c}\text { Initial Rotation } \\
\text { (degrees) }\end{array}$} & \multicolumn{4}{|c|}{ Initial Translation ( $\mathrm{mm})$} \\
\hline & & $1^{\text {st }}$ & $2^{\text {nd }}$ & $3 \pi d$ & $4^{\text {th }}$ \\
\hline \multirow[t]{4}{*}{100} & 0 & 5 & 10 & 20 & 30 \\
\hline & 10 & 5 & 10 & 20 & 30 \\
\hline & 20 & 5 & 10 & 20 & 30 \\
\hline & 30 & 5 & 10 & 20 & 30 \\
\hline \multirow[t]{4}{*}{50} & 0 & 5 & 10 & 20 & 30 \\
\hline & 10 & 5 & 10 & 20 & 30 \\
\hline & 20 & 5 & 10 & 20 & 30 \\
\hline & 30 & 5 & 10 & 20 & 30 \\
\hline \multirow[t]{4}{*}{25} & 0 & 5 & 10 & 20 & 30 \\
\hline & 10 & 5 & 10 & 20 & 30 \\
\hline & 20 & 5 & 10 & 20 & 30 \\
\hline & 30 & 5 & 10 & 20 & 30 \\
\hline
\end{tabular}

\subsection{Results}

The A-ICP and M-ICP registration algorithms were compared the regular ICP registration algorithm. As described above, noise was added to the data model prior to applying the transformation.

To obtain the rotation error, the mean of the absolute difference in rotation values about each axis between the original transformation and the algorithm's transformation were calculated along with the maximum error. To obtain the translation error, the mean distance error (for the 100 trials) between the original 


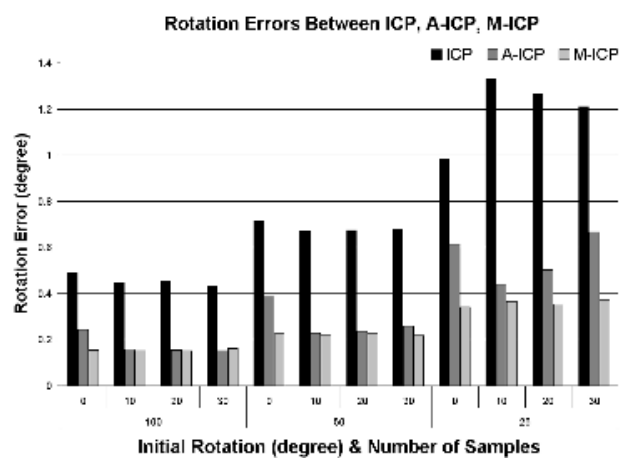

Fig. 4. Evaluation of rotation error for data sets with different number of sampled points and different misalignment. Black bar: ICP, Dark-gray bar: A-ICP, Light-gray bar: M-ICP.

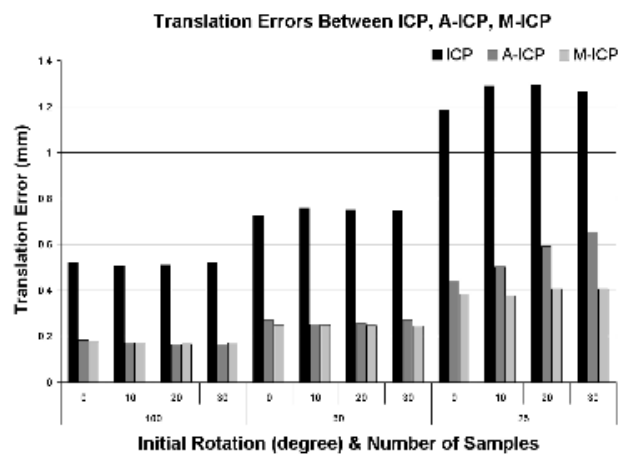

Fig. 5. Evaluation of translation error for data sets with different number of sampled points and different misalignment. Black bar: ICP, Dark-gray bar: A-ICP, Light-gray bar: M-ICP.

transformation and the transformation provided by each algorithm was selected. The results of the experiments are shown in Fig. 4 and 5.

From these results we see that the use of either $4 \mathrm{D}$ registration method has significantly reduced the translation and rotation error in the transformation with the M-ICP method having a slight advantage over the A-ICP method. Considering a typical cardiac environment where the origin of the coordinate system lies in the center of the heart, these methods can result in a target location error (TLE) at the cardiac surface $2-3 \mathrm{~mm}$ less than the standard 3D ICP method. When guiding tools for cardiac ablation procedures based on preoperative images this improvement in accuracy is crucial in the success of the intervention.

For the success criterion of the registration we set the threshold for the mean RMS distance between the heart model and the data model with added noise to be $1.8 \mathrm{~mm}$, to account for the inaccuracies in the localization of the measurements as mentioned in section 3.1. The results in Fig. [6 show that with a significantly 


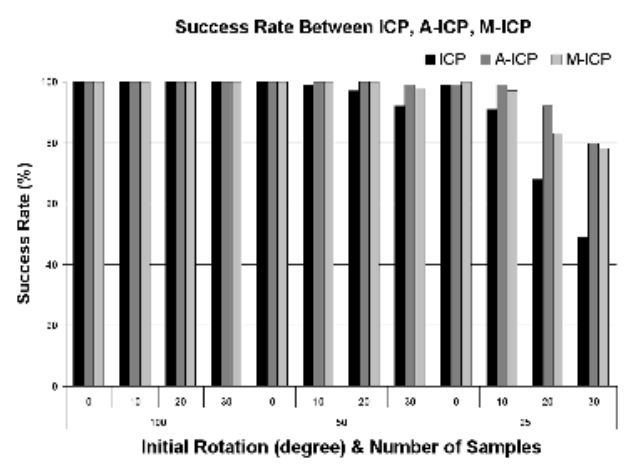

Fig. 6. Evaluation of success rate for data sets with different number of sampled points and different misalignment. Black bar: ICP, Dark-gray bar: A-ICP, Light-gray bar: M-ICP.

large number of data samples the success rate for all methods is $100 \%$. However, when reducing the number of data samples, the success of the 3D ICP drops much more quickly than either of the 4D methods.

Execution time for all the algorithms may vary, however all methods can be parallelized and have very good scalability. This point has practical significance, since the speed with which the registration can be achieved between the acquired EP points and the cardiac model in the operating room is directly related to the overall efficiency of the procedure. For these experiments, the regular ICP registration took approximately 0.2 seconds, while the 4 D registrations took approximately 2 seconds, on a $3.0 \mathrm{GHz}$ Pentium 4 computer with $1 \mathrm{~GB}$ of RAM.

\section{Conclusions}

We have extended the standard 3D ICP method to permit the registration of $4 \mathrm{D}$ datasets. Our experience with two variants of this new approach demonstrated that we can improve both the registration accuracy and the capture range, using the 4D approach rather than employing a series of independent 3D registrations. The anticipated application of these techniques is in our dynamic cardiac mapping environment, where approximately aligned 4D cardiac models and EP data sets are available. We believe that this will permit a more comprehensive cardiac EP mapping and visualization environment than currently existing systems. The advantages of this approach include having a more accurate registration of the tool tracking and image guidance systems, the ability to capture inherent frequency information of the electrocardiogram (an important factor in studying cardiac arrhythmias such as atrial fibrillation), and increasing the intuitiveness of the display by having a dynamic environment where the cardiac map is directly displayed on the patient specific model. Currently, we are conducting phantom and porcine studies to assess the overall accuracy and robustness of our dynamic cardiac mapping environment. 
Acknowledgments. We wish to thank Marcin Wierzbicki for valuable discussions, and funding support from an Ontario Graduate Scholarship (K.W.), CIHR, Canadian Foundation for Innovation and the Ontario Innovation Trust.

\section{References}

1. Kumar, G.A., Maheshwari, A., Thakur, R., Lokhandwala, Y.Y.: Cardiac mapping: Utility or futility? Indian Pacing Electrophysiology Journal 2 (2002) 20-32

2. Sun, Y., Azar, F.S., Xu, C., Hayam, G., Preiss, A., Rahn, N., Sauer, F.: Registration of high-resolution 3D atrial images with electroanatomical cardiac mapping: Evaluation of registration methodology. In: SPIE Medical Imaging 2005: Visualization, Image-Guided Procedures, and Display. Volume 5744. (2005) 299-307

3. Holmes, D.R., Rettmann, M.E., Cameron, B.M., Camp, J.J., Robb, R.A.: Virtual cardioscopy: Interactive endocardial visualization to guide RF cardiac ablation. In: SPIE Medical Imaging 2006. Volume 6143. (2006)

4. Wierzbicki, M., Drangova, M., Guiraudon, G., Peters, T.: Validation of dynamic heart models obtained using non-linear registration for virtual reality training, planning, and guidance of minimally invasive cardiac surgeries. Medical Image Analysis 8 (2004) 387-401

5. Besl, P.J., McKay, N.D.: A method for registration of 3-D shapes. IEEE Transactions on Pattern Analysis and Machine Intelligence 14 (1992) 239-256

6. Horn, B.K.P.: Closed-form solution of absolute orientation using unit quaternions. Journal of the Optical Society of America 4 (1987) 629-642

7. Su, Y., Holmes, D.R., Rettmann, M.E., Robb, R.A.: A piecewise function-tostructure registration algorithm for image guided cardiac catheter ablation. In: SPIE Medical Imaging 2006. Volume 6141. (2006)

8. Estepar, R.S.J., Brun, A., Westin, C.F.: Robust generalized total least squares iterative closest point registration. In: MICCAI 2004, LNCS. Volume 3216. (2004) $234-241$

9. Chetverikov, D., Svirko, D., Stepanov, D., Krsek, P.: The trimmed iterative closest point algorithm. In: International Conference on Pattern Recognition. Volume 3. (2002) 545-548

10. Gramkow, C.: On averaging rotations. Journal of Mathematical Imaging and Vision 15 (2001) 7-16

11. Moore, J., Drangova, M., Wierzbicki, M., Barron, J., Peters, T.: A high resolution dynamic heart model based on averaged MRI data. In: MICCAI 2003, LNCS. Volume 2878. (2003) 549-555 